\title{
Feedback Following the Creation of Epijournal de Géométrie Algébrique
}

Pierre-Emmanuel Chaput (Université de Lorraine, Vandœuvre-lès-Nancy, France), Benoît Claudon (Université de Rennes, France), Damien Mégy (Université de Lorraine, Vandœuvre-lès-Nancy, France), Lucas Fresse (Université de Lorraine, Vandœuvre-lès-Nancy, France), Alain Genestier (Université de Lorraine, Vandœuvre-lès-Nancy, France), Arvid Perego (Université de Lorraine, Vandœuvre-lès-Nancy, France), Matei Toma (Université de Lorraine, Vandœurre-lès-Nancy, France) $)^{\star}$

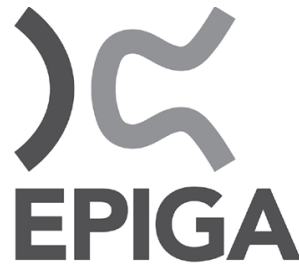

A new overlay journal, the Épijournal de Géométrie Algébrique, was recently launched. In this note, the launch serves as the starting point of a discussion of overlay journals in general and the Épisciences platform ${ }^{1}$ in particular. We then report in more detail on the Épijournal de Géométrie Algébrique.

\section{Overlay journals: a new model of scientific pub- lication}

Let us start with a reminder of what an overlay journal (or épijournal in French) is. It is an open-access scientific journal that is constructed by adding extra structure on top of one (or more) pre-existing open-access archives.

Overlay journals follow exactly the same peer-review process as a traditional mathematical journal. The articles are generally electronically published and also remain on the preprint server where they were initially published.

This description justifies the term "open access". By its very nature, an overlay journal provides open access to all published articles. In the next section, we discuss the economic model of one overlay platform - Épisciences.

\section{The Épisciences platform}

An overlay journal is an extra layer on top of one or more open archives, with which it usually connects via a web platform. Épisciences is a web platform that has been created to host overlay journals. It currently hosts journals in mathematics and computer science.

This platform is backed by the following open archives for mathematics - arXiv and Hyper Articles en Ligne (HAL) - and is a project of the CCSD (Centre for Direct Scientific Communication). Overlay journals hosted on Épisciences are economically ethical since they do not demand publication fees and their costs are paid by public institutions.

* The authors gratefully acknowledge Catriona Maclean for her help in writing this text.

1 We also suggest reading https:/gowers.wordpress.com/2013/ 01/16/why-ive-also-joined-the-good-guys/.

2 Overlay journals are not only for mathematicians and computer scientists (see, for example, http://www.nature.com/ news/open-journals-that-piggyback-on-arxiv-gather-momentum-1.19102).
The platform offers a web infrastructure for managing editorial flow. Note that this platform does not only host new journals: existing journals may decide to switch to overlay journals and transition into the Épisciences fold.

At the time of writing (September 2017), the Épisciences platform hosts two overlay journals in mathematics (including the Épijournal de Géométrie Algébrique), five in computer science and three in the humanities.

\section{Épijournal de Géométrie Algébrique}

Observation of difficulties of scientific publishing led the authors to the idea of using the Épisciences platform to launch a new mathematical journal. We found it easier to create a specialized journal rather than a general one, particularly for setting up an editorial board, and we soon decided that the scope of our journal would be algebraic geometry.

\section{Starting the project}

Our first tasks were to find a name, form the editorial board and fix our operating rules. We then gathered this information and passed it onto the épiMaths committee. ${ }^{3}$ The role of this committee is to encourage the creation of new overlay journals and study the applications made for that purpose. Our application was accepted on December 2015.

From January to June 2016, we learned how to use the Épisciences platform and started testing it. During that time, we made some requests for new developments to make the platform compatible with our operating rules.

Prior to the launch of our journal, we also had to create website content, keep the editors informed of the platform operating procedures and start prospecting for possible submissions.

\section{About the journal}

As the name suggests, Épijournal de Géométrie Algébrique exists to publish original articles in algebraic geometry in a broad sense, from arithmetic geometry to the study of compact Kähler varieties through to the theory of algebraic groups and their representations.

The composition of the editorial board (and more details of the journal) can be found at:

https://epiga.episciences.org/.

\footnotetext{
The composition of this committee is available at http://episciences.org/page/epimath.
} 
One of the editors ${ }^{4}$ has the special role of coordinator. His role is to assign an editor to each article and to facilitate discussions on the status of a submitted article. Acceptance or rejection decisions are made conjointly by the whole board.

The authors of this note are members of the monitoring committee of the journal. The role of this committee is to ensure that the logistics of the journal work properly (e.g. setting the journal's website, testing the platform interface and developing a LaTeX style journal sheet). Together with the editors, we promote this fledgling journal through relevant mailing lists and by direct article solicitation.

\section{The first volume}

The first volume has recently been published and its content can be seen at:

https://epiga.episciences.org/volume/view/id/267.

For the time being, it consists of nine articles but other articles accepted in the current year will be added to this first volume. Subsequently, the journal will publish one volume per year.

\section{Conclusion}

We have just talked about the conclusion, i.e. soliciting valuable submissions is a task of importance. Any new submission is welcome and we hope that this article will have encouraged this!

Perennial and institutional alternatives to the economic models offered by commercial publishers come in different forms: the Épisciences project is one of

\footnotetext{
4 Currently Michel Brion.
}

those. Let us grasp and expand the opportunities so that researchers willing to publish their work in economically virtuous journals have a wider and wider choice.

Pierre-Emmanuel Chaput works at the Elie Cartan Institute (IECL), Nancy, France. His research focuses on the interplay between algebraic groups, representation theory, and algebraic geometry.

Benoit Claudon works at the Mathematic Institute of Rennes (IRMAR), France. His field of interest is complex algebraic geometry (classification of smooth projective varieties and compact Kähler manifolds).

Lucas Fresse works at the Elie Cartan Institute (IECL), Nancy, France. His research topics concern geometric representation theory and Lie theory.

Alain Genestier works at the Elie Cartan Institute (IECL), Nancy, France. His research focuses on arithmetic algebraic geometry, with a focus on the consequences in the local Langlands program of the bad reduction behavior of Shimura varieties and shtuka classifying spaces.

Damien Mégy works at the Elie Cartan Institute (IECL), Nancy, France. His field of interest is complex algebraic geometry with a focus on Hodge theory.

Arvid Perego works at the Elie Cartan Institute (IECL), Nancy, France. His research focuses on hyperkahler manifolds and moduli spaces of semi-stable sheaves (or complexes of sheaves) on surfaces.

Matei Toma works at the Elie Cartan Institute (IECL), Nancy, France. His field of research is Complex Geometry. 